\title{
Optimal Positioning of Distributed Generator using Hybrid Optimization algorithm in Radial Distribution System
}

\author{
Ravikumar S \\ Department of EEE \\ Swami Ramanand Theerth Marathwada University \\ Nanded, Maharashtra, India \\ raviskumar197@gmail.com
}

\section{Deepak R}

Department of EEE

College of Engineering, Adoor,

Manakala, Kerala, India

\author{
Vennila H \\ Department of EEE \\ Amrita college of engineering and Technology, \\ Eracha Kulam, Tamil Nadu, India
}

\begin{abstract}
In distribution systems, the distributed generator is renowned as a feasible solution in order to control the line losses, voltage stability, and bus voltage, etc. This paper concentrates on an advanced technique in order to position the distributed generator. It is exploited for minimization of the energy loss and active power loss for the distribution lines when concerning the voltage stability index and bus voltage. Based on the optimal positioning and sizing of the DGs, the optimization is performed. Here, a hybrid ABC and Bat Algorithm (HABC-BA) is presented to solve the optimal distributed generators allocation issue of distribution networks. The proposed HABC-BA method experimented on standard 33-bus, 69bus in radial distribution networks to examine the possibility and efficiency. Hence, the experimental analysis shows that placement of distributed generators in the optimal position can extensively minimize the power loss in a DS Finally, the proposed method is compared with other meta-heuristic approaches such as GWO, WOA and PSO and the analysis exhibit that the proposed technique has the capability to find enhanced quality solutions.
\end{abstract}

Keywords: Distributed Generators; Optimal Size; Power Loss; Optimal Location; Optimization Algorithm

\begin{tabular}{ll}
\hline Abbreviations & Descriptions \\
\hline RDS & Radial Distribution Systems \\
GA & Genetic Algorithm \\
PSO & Particle Swarm Optimization \\
ACO & Ant Colony Optimizer \\
HS & Harmony Search \\
OPAS & Optimal Placement and Sizing \\
MICP & Mixed Integer Conic Programming \\
ESD & Energy Storage Devices \\
FF & Firefly \\
APF & Active Power Filter \\
DS & Distribution System \\
ABC & Artificial Bee Colony \\
REG & Renewable Energy Generator \\
CTLBO & Comprehensive Teaching Learning-Based Optimization \\
DN & Distribution Network \\
WOA & Whale Optimization Algorithm \\
VSI & Voltage Stability Index \\
DER & Distributed Energy Resources \\
GWO & Grey Wolf Optimizer \\
DS & Distribution System \\
NLPCI & New Nonlinear Load Position based APF Current Injection \\
OADG & Optimal Allocation of Distributed Generators \\
CBN & Critical Bus number \\
CBV & Critical Bus Voltage \\
IDSA & Improved Differential Search Algorithm \\
SFSA & Stochastic Fractal Search Algorithm \\
\hline
\end{tabular}




\section{Introduction}

Nowadays, an extensive scope of experimentation is taking place in the DS because it is the ultimate connection among immensity consumers and the power system. Basically, at high currents and low voltage, the DS works that outcome in a huge number of penurious voltage profiles and loss of power, as well as while comparing with the transmission system. Moreover, it is well-known that the approximate share of the distribution losses in total power generated is $13 \%$ of the research analysis. Due to the frequent increases in the demand for electricity tends to extend augmentation in voltage drop and power. This kind of maximum power losses restricts the capacity line and penurious voltage profile leads the system voltage instability [17]. Hence, the main agitates is not merely regarding the enhancements of technical features but as well as to encounter the future demand of electricity for the conventional DS by conserving the voltage stability and the capacity line.

Generally, distributed generation is considered as the small-scale scattered origin of electric power that positioned close to the load's existence overhauled. Various distributed generation applications are in implementation namely small hydro, wind turbines, biomass, photovoltaic cells, fuel cells, and microturbines. In the DSs [18], the distributed generations have been broadly worked that taken into consideration of procedural benefits for the power grids like minimization of power loss, voltage profile enhancement and security of the system, enhancing consistency, and augmentation of coherence energy.

In power systems, due to the superior consequences of distributed generations, positioning them is high and high well-liked in DSs [19]. In DSs incorporating distributed generations offers few advantages like improvement of voltage profiles, minimizing power losses, minimization of environmental pollution and enhancement of power quality. Additionally, an appropriate distributed generation applications aids to minimize the pressure on improving the fuel, gird as well as costs of operating. By determining the framework of the power grid as well as the applications of distributed generations, the effect of distributed generations is not similar [20]. Nevertheless, the unsuitable position of distributed generations might augment losses of the system as well as appropriate costs. Hence, it is significant to discover the optimal sizing and positioning of DG to attain the aforesaid objectives in DSs. In addition, the distributed generation designing in RDS must contemplate various factors comprise positioning and sizing, amount of distributed generations, as well as exploited application. Amid the aforesaid factors, in distributed generation planning the one of the most important factor are the optimal positioning and sizing of distributed generations [16] [21].

This is not merely an issue but as well as a chance for researchers to discover techniques in order to deal with this issue [22]. Therefore, several techniques were presented to resolve these issues. Moreover, these approaches can be categorized into types such as existing, intelligent and hybrid techniques. Additionally, researchers have examined several optimization techniques in order to find the optimal positioning and sizing for distribution generators like PSO [13], GA [15] as well as its enhanced versions, ACO, HS approach, FF [14]. Last decades, artificial intelligence-based techniques have been extensively exploited in order to solve the optimal positioning and sizing issue with attained propitious outcomes. Most of these techniques have various fine points namely the capability to find out a near-optimal solution for nonlinear issues and the potential fin order to deal with the large-scale complex systems.

The main objective of the paper is to introduce the Hybrid ABC-BA optimization technique to the energy loss and active power loss for the distribution lines when concerning the voltage stability index and bus voltage.

\section{Literature Review}

In 2019, Satish Kumar Injeti [1], worked on optimal positioning and sizing selections for distributed generations in RDS. It was exploited to reduce the losses as well as the operating cost and enhancement of voltage profile. They have presented IDSA, which was exploited to resolve the optimization issue through Pareto optimal technique by representing financial and technical advantages of distributed generations as objectives.

In 2018, Tri Phuoc Nguyen, Dieu Ngoc Vo [2], proposed an application for a new SFSA in order to solve the OADG issue in RDSs. The proposed technique was a novel effectual meta-heuristic technique, which was enthused by developing a procedure of nature and it was exploited by a mathematical notation termed as a fractal to optimization issues. The benefits of the presented approach were uncomplicated execution as well as some control parameters. Moreover, the technique was presented in order to pact with the OADG issue.

In 2019, Ashokkumar Lakum and Vasundhara Mahajan [3], discussed an effect of Distributed Generators diffusion on the OPAS of APF. Here, they have proposed an NLPCI approach in order to 
place the possible buses for the APF positioning in attendance of Distributed Generators and nonlinear load. Moreover, the GWO was exploited to distinguish the optimal positioning of APF.

In 2019, Juan M. Home-Ortiza et al [4], presented a MICP framework to discover the optimal positioning and sizing of distributed generators against a multistage planning horizon in RDS. The presented model planning concentrates on the optimal positioning and sizing of photovoltaic panels, wind and gas turbines, and ESD. Naturally, demands on electricity and renewable energy sources were focused on uncertainty. In decision making, the proposed framework was comprehensive into a dualphase stochastic programming framework in order to hold such probabilistic circumstances.

In 2018, Imran Ahmad Quadri et al [5], proposed a CTLBO method to allocate the distributed generations in RDS to enhance the minimization of network loss, energy savings, and voltage profile. Moreover, the developed method has the ability to hold mixed-integer variables, which were the parameter self-governing as well as it has resistance to local extrema trappings. In standard mathematical benchmark functions, the efficiency of the developed method was examined.

In 2017, Haifeng HONG et al [6], proposed a directed graph-based technique by representing distributed generations for the reconfiguration of the DN. Here, dual reconfiguration conditions were represented such as operation mode modification in terms of the objective of reducing active power loss and service restoration in terms of the objective of increasing loads restored. The aforesaid dual conditions were framed as a mixed-integer quadratic and linear programming issue.

In 2016, Qiuye Sun et al.[7], presented a novel distributed coordinated controller in a combination of a multiagent-based consensus approach. It was exploited in the DGs for the energy internet. Subsequently, the decomposed chores, frameworks, as well as the flow of the information for the presented technique were examined. The presented coordinated controller positioned among the Energy Internet remains amplitude compromise and voltage angles when offering precise power-sharing as well as reducing circulating currents.

In 2019, Kamakshi Prashadini Swain and Mala De [8] presented a voltage control technique for smart DS exploiting a new electrical immediacy index. This index was self-governing for the load dynamics system. On the basis of the electrical proximity in the control units this technique works, by means of DER and flexible loads. By voltage deviation, the role of these units was computed for different time steps spanning against the day on the basis of the proximity of control units.

\section{Mathematical Formulation}

\subsection{Objective Model}

In RDS, the main aim of the positioning of distributed generation is to reduce the losses of the network power when fulfilling particular operating constraints. Here, the objective model of power loss minimization is described in eq. (1). Here, $\mathrm{P}_{\text {loss }}$ represents the real power loss of the DN and it is denoted in eq. (2) [9].

$$
\begin{aligned}
& \mathrm{F}=\min \left(\mathrm{P}_{\text {loss }}\right) \\
& \left.\mathrm{P}_{\text {loss }}=\sum_{\mathrm{i}=1}^{\mathrm{n}_{\mathrm{b}}} \sum_{\mathrm{j}=1}^{\mathrm{n}_{\mathrm{b}}} \frac{\mathrm{S}_{\mathrm{i}, \mathrm{j}}}{\mathrm{V}_{\mathrm{i}} \mathrm{V}_{\mathrm{j}}} \cos \partial_{\mathrm{i}}-\partial_{\mathrm{j}}\right)\left(\mathrm{P}_{\mathrm{i}} \mathrm{P}_{\mathrm{j}}+\mathrm{Q}_{\mathrm{i}} \mathrm{Q}_{\mathrm{j}}\right)+\frac{\mathrm{s}_{\mathrm{i}, \mathrm{j}}}{\mathrm{V}_{\mathrm{i}} \mathrm{V}_{\mathrm{j}}} \sin \left(\partial_{\mathrm{i}}-\partial_{\mathrm{j}}\right)\left(\mathrm{Q}_{\mathrm{i}} \mathrm{P}_{\mathrm{j}}+\mathrm{P}_{\mathrm{i}} \mathrm{Q}_{\mathrm{j}}\right)
\end{aligned}
$$

In eq. (2), $P_{i} Q_{i}$ represents the net active power and $P_{j} Q_{j}$ represents the reactive power, at the $\mathrm{i}^{\text {th }}$ bus, $\mathrm{s}_{\mathrm{i}, \mathrm{j}}$ represents the distribution line resistance-associated among the $\mathrm{i}^{\text {th }}$ and the $\mathrm{j}^{\text {th }}$ bus, $V_{i}$ represents the voltages of bus at the $i^{\text {th }}$ and the $V_{j}$ represents the voltages of bus $j^{\text {th }}$ bus; $n^{b}$ represents the number of buses in the DN, $\partial_{i}$ and $\partial_{\mathrm{j}}$ represents the voltage phase angle at the $\mathrm{i}^{\text {th }}$ and the $\mathrm{j}^{\text {th }}$ bus, correspondingly.

By maximizing the loading as well as utilization of the conventional power structure, the probability incidence of voltage collapse is notably higher than previous incidence and the nodes recognition. However, this is prone to the voltage fluctuations; it attracts more interest in the DSs. In a secure and safe way to operate the power system, all insecure operating conditions need to be recognized well in order to precede the ease remedial measures to conquer the risk of probable voltage collapse. Hence, in a secure and safe way to function the DN, the VSI needs to be within a definite limit. Eq. (3) denotes the VSI of radial distribution [10].

$$
\operatorname{VSI}_{\mathrm{i}}=\left|\mathrm{V}_{\mathrm{j}}\right|^{4}-\left.4\left[\mathrm{P}_{\mathrm{i}} \mathrm{s}_{\mathrm{i}, \mathrm{j}}+\mathrm{Q}_{\mathrm{i}} \mathrm{x}_{\mathrm{i}, \mathrm{j}}\right] \mathrm{V}_{\mathrm{j}}\right|^{2}-4\left[\mathrm{P}_{\mathrm{i}} \mathrm{x}_{\mathrm{i}, \mathrm{j}}-\mathrm{Q}_{\mathrm{i}} \mathrm{s}_{\mathrm{i}, \mathrm{j}}\right]
$$


where, $i=2,3,4, \ldots j_{b}$

In eq. (3), $\mathrm{VSI}_{\mathrm{i}}$ represents the voltage stability index of the $\mathrm{i}^{\text {th }}$ bus; $\mathrm{x}_{\mathrm{i}, \mathrm{j}}$ represents the distribution line reactance -associated among the $\mathrm{i}^{\text {th }}$ and the $\mathrm{j}^{\text {th }}$ bus; $\mathrm{s}_{\mathrm{i}, \mathrm{j}}$ represents the distribution line resistanceassociated among the $\mathrm{i}^{\text {th }}$ and the $\mathrm{j}^{\text {th }}$ bus.

In this paper, the main objective is to minimize the energy loss for the positioning of the Renewable Energy Generator (REG) on the basis of the distributed generation units. For developing REGs, the energy loss minimization for the RDS is considered as a significant feature. Over a year, the total energy losses minimization of the DS is defined in eq. (4).

$$
\min \mathrm{E}_{\text {loss }}=\operatorname{Min} 365 \sum_{\mathrm{i}=1}^{24} \mathrm{P}_{\text {Loss }}(\mathrm{t}) \times \Delta \mathrm{t}
$$

where $\mathrm{E}_{\text {loss }}$ represents the energy loss more than a year; $\mathrm{P}_{\mathrm{Loss}}(\mathrm{t})$ represents the active power loss at the $\mathrm{t}^{\text {th }}$ time interval; $\Delta t$ represents the time duration in each step.

\subsection{Constraints}

The aforesaid objective functions are referred to like the below constraints:

(a) Constraints of power balance

$$
\mathrm{P}_{\mathrm{i}}+\mathrm{nQ}_{\mathrm{i}}=\mathrm{V}_{\mathrm{i}} \mathrm{M}_{\mathrm{i}}^{*}
$$

In eq. (5), $\mathrm{M}_{\mathrm{i}}$ indicates the current at the $\mathrm{i}^{\text {th }}$ node.

(b) Constraints of voltage limit

The constraint of voltage should be considered by stating the lower and upper limits of voltage deviation at the nodes of the DS and it is represented in eq. (6). Here, $V_{i}^{\max }$ represents the upper limit of the bus voltage and $V_{i}^{\min }$ represents the lower limit of the bus voltage correspondingly.

$$
\mathrm{V}_{\mathrm{i}}^{\min }<\mathrm{V}_{\mathrm{i}}<\mathrm{V}_{\mathrm{i}}^{\max }
$$

(c) Constraints of thermal limit

$$
\mathrm{M}_{\mathrm{i}, \mathrm{j}} \leq \mathrm{M}_{\mathrm{i}, \mathrm{j}}^{\max }
$$

In eq. (7), $\mathrm{M}_{\mathrm{i}, \mathrm{j}}^{\max }$ represents the maximum loading of the distribution line-associated among the $\mathrm{i}^{\text {th }}$ and the $\mathrm{j}^{\text {th }}$ bus; $\mathrm{M}_{\mathrm{i}, \mathrm{j}}$ represents the current flowing by the branch associated among the $\mathrm{i}^{\text {th }}$ and the $\mathrm{j}^{\text {th }}$ bus branch.

(d) Constraints of real power limit

$$
\mathrm{P}_{\mathrm{i}, \text { min }}^{\mathrm{DG}}<\mathrm{P}_{\mathrm{i}}^{\mathrm{DG}}<\mathrm{P}_{\mathrm{i}, \text { max }}^{\mathrm{DG}}
$$

where $\mathrm{P}_{\mathrm{i}, \text { min }}^{\mathrm{DG}}$ and $\mathrm{P}_{\mathrm{i}, \max }^{\mathrm{DG}}$ represents the lower and upper limits, correspondingly, $\mathrm{P}_{\mathrm{i}}^{\mathrm{DG}}$ represents the active power of the $\mathrm{i}^{\text {th }} \mathrm{DG}$.

(e) Constraints of reactive power limit

$$
\mathrm{Q}_{\mathrm{i}, \text { min }}^{\mathrm{DG}}<\mathrm{Q}_{\mathrm{i}}^{\mathrm{DG}}<\mathrm{Q}_{\mathrm{i}, \max }^{\mathrm{DG}}
$$

where, $Q_{i, \text { min }}^{\mathrm{DG}}$ and $\mathrm{Q}_{\mathrm{i}, \max }^{\mathrm{DG}}$ represents the minimum and maximum permissible reactive power, correspondingly, $\mathrm{Q}_{\mathrm{i}}^{\mathrm{DG}}$ represents the active power of the $i^{\text {th }} \mathrm{DG}$.

\section{Hybrid optimization Algorithm for Optimal Positioning of Distributed Generators}

\subsection{Conventional Bat Algorithm}

Bat algorithm refers to a novel approach showing to provide an enhanced solution than numerous wellliked conventional and heuristic methods [11] and it is exploited to solve the complex engineering issues. This technique works on the basis of the echolocation of microbats. Moreover, the echolocation refers the attractive sonar wave that produced by the microbats; it assists them to identify the prey in a few supernatural manners, the bats have the ability to distinguish the various types of obstructions or hazard on the technique in the direction of the prey in the whole darkness.

The bats produce noisy ultrasonic sound waves and pay attention to the echo, which reproduces back from the nearby objects. The bat algorithm exploits a few admires rules for ease. 
a) In a random way, the Bats fly with the velocity $\mathrm{u}_{\mathrm{i}}$ at the location $\mathrm{y}_{\mathrm{i}}$. It has the ability to regulate their generated pulses frequency and regulate the pulse emission rate $a \in[0,1]$, based on the nearness of their objective.

b) To regulate the noise, there are so many methods. For ease, the noise is presumed to deviate from a positive great $B_{0}$ to the least constant value that is indicated by $B_{\min }$.

In [11], the eq. (10), (11) and (12), represents the virtual bat movement is simulated.

$$
\begin{aligned}
\mathrm{f}_{\mathrm{i}} & =\mathrm{f}_{\min }+\left(\mathrm{f}_{\max }-\mathrm{f}_{\min }\right) * \alpha \\
\mathrm{u}_{\mathrm{i}}^{\mathrm{t}} & =\mathrm{u}_{\mathrm{i}}^{\mathrm{t}-1}+\left(\mathrm{y}_{\mathrm{i}}^{\mathrm{t}-1}-\mathrm{y}_{\text {best }}\right) * \mathrm{f} \\
\mathrm{x}_{\mathrm{i}}^{\mathrm{t}} & =\mathrm{u}_{\mathrm{i}}^{\mathrm{t}-1}+\mathrm{u}_{\mathrm{i}}^{\mathrm{t}}
\end{aligned}
$$

In eq. (10), $f$ represents the frequency, exploited by the bat looking for its prey; $f_{\min }$ indicates the minimum value and $f_{\max }$ indicates utmost value correspondingly. The $y_{i}$ represents the position of the $\mathrm{i}^{\text {th }}$ bat in the solution space, $u_{i}$ indicates the bat velocity, $\mathrm{f}$ denotes the current iteration, $\alpha$ represents the arbitrary vector that is haggard from a uniform distribution, and $\alpha \in[0,1]$, and $\mathrm{y}_{\text {best }}$ refers the global near-optimal solution create hitherto against the entire population. Moreover, in the procedure, the pulse rate emission from the bat is represented as one of the tasks. The micro-bat produces the echo and alters the frequency that depends on the nearness of their objective. The rate of pulse emission is represented using $\mathrm{e}_{\mathrm{i}}$, and $e_{i} \in[0,1]$, the suffix $i$ represents the $\mathrm{i}^{\mathrm{t}}$ bat. An arbitrary number is produced that is compared with $\mathrm{e}_{\mathrm{i}}$ in every iteration. If the arbitrary number is higher than $e_{i}$, a local search approach, like, arbitrary walk, is exploded. Based on eq. (13) a new solution of the bat is created.

$$
\mathrm{y}_{\text {new }}=\mathrm{y}_{\text {old }}+\phi \mathrm{B}^{\mathrm{t}}
$$

In eq. (13), $\phi$ represents a random number and $\phi \in[-1,1]$, and $\mathrm{B}^{\mathrm{t}}$ indicates the average noise of all bats at the current time step. Subsequent to locations updating of the bats, the sound $B_{i}$ and the rate of pulse emission $e_{i}$ are also updated only while the global near-optimal solution is updated and the randomly created number is lesser than $B_{i}$. Using eq. (14) and (15), the updating of $B_{i}$ and $e_{i}$ are operated. Here, $\beta$ and $\eta$ represents the constants. In [11], $\beta$ and $\eta$ is set as 0.9 , which is exploited for the effortlessness.

$$
\begin{gathered}
\mathrm{B}_{\mathrm{i}}^{\mathrm{t}+1}=\beta \mathrm{B}_{\mathrm{i}}^{\mathrm{t}} \\
\mathrm{e}_{\mathrm{i}}^{\mathrm{t}+1}=\mathrm{e}_{\mathrm{i}}^{0}\left[1-\mathrm{a}^{-\eta t} \mid\right.
\end{gathered}
$$

\subsection{Conventional Artificial Bee Colony Algorithm}

The ABC technique [12] works on the basis of examining the behaviors of real bees. Moreover, it describes the nectar finding and information sharing of food sources to the bees in the nest. Here, the bees are classified into three types such as the onlooker, employed bee, and the scout. In the optimization procedure, every type of bees plays dissimilar and significant functions. For instance, the employed bee remains on a food source that indicates a stain in the solution space, as well as offers the coordination of the onlookers in the hive for the position. The onlooker bee obtains the positions of the food sources and chooses one of the food sources to meet the nectar. To search for new food sources, the scout bee moves in the solution space. The procedure of ABC optimization is stated below:

a) In the initialization process, scatter $s_{e}$ populations percentage into the solution space in a random manner and after that their fitness values is computed, which refers to the nectar amounts. Here, $s_{\mathrm{e}}$ indicates the employed bees ratio of the total population. In solution space, once these populations are located they are referred to as the employed bees. The employed bee's fitness value is assessed to considering in their nectar amount.

$$
\mathrm{PS}_{\mathrm{i}}=\frac{\mathrm{F}\left(\phi_{\mathrm{i}}\right)}{\sum_{\mathrm{k}=1}^{\mathrm{e}_{\mathrm{b}}} \mathrm{F}\left(\phi_{\mathrm{i}}\right)}
$$

b) Move the Onlookers: Compute the probability of a food source selection is computed using eq. (16), where $\phi_{i}$ represents the location of the $i^{\text {th }}$ employed bee, The fitness function is denoted by $F\left(\phi_{i}\right), e_{b}$ indicates the number of employed bees, and $\mathrm{PS}_{\mathrm{i}}$ represents the selection probability of the $\mathrm{i}^{\text {th }}$ employed bee. For every onlooker bees, the selection of roulette wheel technique is exploited to food source selection in order to move and after that decide the nectar amounts of them. Using eq. (17), the onlookers are 
moved, where $y_{i}$ indicates the location of the $i^{\text {th }}$ onlooker bee, $t$ indicates the iteration number, $\phi_{i}$ represents the arbitrarily employed bee selection, $j$ indicates the solution dimension, and $\theta(\cdot)$ creates a random series variable in the range from -1 to 1 .

$$
\mathrm{y}_{\mathrm{ij}}(\mathrm{t}+1)=\phi_{\mathrm{ij}}(\mathrm{t})+\theta\left(\phi_{\mathrm{ij}}(\mathrm{t})-\phi_{\mathrm{kj}}(\mathrm{t})\right)
$$

c) Updating the optimal food source establishes until now: The optimal fitness value, as well as the location, is memorized that are established by the bees.

d) Move the Scouts: If the fitness values of the employed bees do not enhance using a continuous encoded number of iterations that is termed as "Limit". The aforesaid food sources are neglected, and these employed bees turn out to be the scouts. Using eq. (18), the scouts are moved, e represents an arbitrary number and $e \in$ range from 0 to 1 .

$$
\phi_{\mathrm{ij}}=\phi_{\mathrm{j} \min }+\mathrm{e} \times\left(\phi_{\mathrm{j} \max }-\phi_{\mathrm{j} \min }\right)
$$

e) Termination: If the termination state is fulfilled, finish the program as well as output the outcomes; else go back to process state in (b).

\subsection{Proposed Hybrid ABC-BA Algorithm}

The proposed technique is structured by hybridization between two techniques such as $\mathrm{ABC}$ and $\mathrm{BA}$ algorithm. According to the fitness evaluation of one technique, this hybridization works, by replacing the frailer individuals with powerful individuals from other technique in series processing for swarm intelligent techniques. In a series structure, various groups are produced from dividing the population into subpopulations to make the hybridizing technique. In regular iterations, each of the subpopulations develops separately. While the scheme is activated, they merely swap information among populations. It outcomes in captivating benefit of the individual strengths for each kind of technique, restoring the frailer individuals with the enhanced one from other, the population size minimization for each population as well as the advantage of collaboration is attained.

By optimizing separately, each technique develops, that is the BA has possessed its bats and close to the optimal solution to put backs artificial agents of $\mathrm{ABC}$ worst as well as not the close-optimal solution. Conversely, the artificial agents enhanced ABC are exploited to put back the worse bats of Bat Algorithm subsequent to running $N_{i}$ iterations. The total iteration comprises $\mathrm{N}$ number of communication, where $\mathrm{N}=\left\{\mathrm{N}_{1}, 2 \mathrm{~N}_{1}, 3 \mathrm{~N}_{1} \ldots\right\}$. In Bat Algorithm, the bats do not have an idea in the solution space about the survival of artificial bees of ABC.

In the proposed algorithm, let us consider $\mathrm{P}$ be the number of population, and $\mathrm{P}_{1}, \mathrm{P}_{2}$ be the number of population of proposed algorithm correspondingly, where $\mathrm{P}_{1}=\mathrm{P}_{2}=\mathrm{P} / 2$. If $t \cap N \neq \sigma$, m agents with the top $\mathrm{m}$ fitness in $\mathrm{P}_{1}$ will be copied to $\mathrm{P}_{2}$ to put back the similar number of agents with the worst fitness, where $t$ indicates the current iteration count, $\mathrm{N}_{\mathrm{i}}$ and $\mathrm{m}$ are the predefined constants.

a) Initialization: For both $\mathrm{ABC}$ and Bat algorithm generate populations. By $\mathrm{BA}$ and $\mathrm{ABC}$, initialize each population independently. For executing the communication scheme, the iteration set $N$ is defined. The $\mathrm{P}_{1}, \mathrm{P}_{2}$ bats as well as artificial agents $\mathrm{e}_{\mathrm{b}, \mathrm{j}}^{\mathrm{T}}$ and $\mathrm{Y}_{\mathrm{i}, \mathrm{j}}^{\mathrm{T}}$ for populations of $\mathrm{ABC}$ and $\mathrm{BA}$ correspondingly, $\mathrm{i}=0,1, \ldots . \mathrm{P}, \mathrm{j}=0,1$, and $t$ indicates the current iteration number. Set $\mathrm{t}=1$.

b) Evaluation: In each population, for both the $\mathrm{ABC}$ and $\mathrm{BA}$ algorithm calculate the value of $\mathrm{f}_{1}\left(\mathrm{e}_{\mathrm{b}_{\mathrm{i}, \mathrm{j}}}^{\mathrm{T}}\right), \mathrm{f}_{2}\left(\mathrm{Y}_{\mathrm{i}, \mathrm{j}}^{\mathrm{T}}\right)$. By both $\mathrm{ABC}$ and $\mathrm{BA}$ optimization, the population's evolvement is executed independently.

c) Update: By eq. (10), (11) and (12), the locations and the velocity of Bat are updated. The location and the optimal fitness value are memorized that are established by the bees exploited by eq. (16), (17) and (18).

d) Communication scheme: Amid all the individual, the optimal bats of BA algorithm population is migrated, copy $\mathrm{m}$ individuals with the top $\mathrm{m}$ fitness in $\mathrm{P}_{1}$ replacing the subordinate agents in $\mathrm{P}_{2}$ of ABC's population and for each population in each $\mathrm{N}_{1}$ iteration are updated.

On the other hand, among all the individual of ABC's population, the optimal artificial agents are migrated, copy $\mathrm{m}$ agents with the top $\mathrm{m}$ fitness in $\mathrm{P}_{2}$ putting back the subordinate bats in $\mathrm{P}_{1}$ and for each population every $\mathrm{N}_{1}$ iteration are updated.

5. Termination: Repeat the process stated from (b) to (e) till the predefined function value accomplished or the utmost number of iterations is achieved. The optimal value of the function is recorded $\mathrm{f}\left(\mathrm{e}_{\mathrm{b}}^{\mathrm{T}}\right)$ and the optimal bat location between all the bats $\mathrm{e}_{\mathrm{b}}^{\mathrm{T}}$. The optimal function value is 
recorded $\mathrm{f}\left(\mathrm{Y}^{\mathrm{T}}\right)$ and the optimal food source between all the agents $\mathrm{Y}^{\mathrm{T}}$. Fig. 1 demonstrates the diagrammatic representation of proposed HABC-BA algorithm.

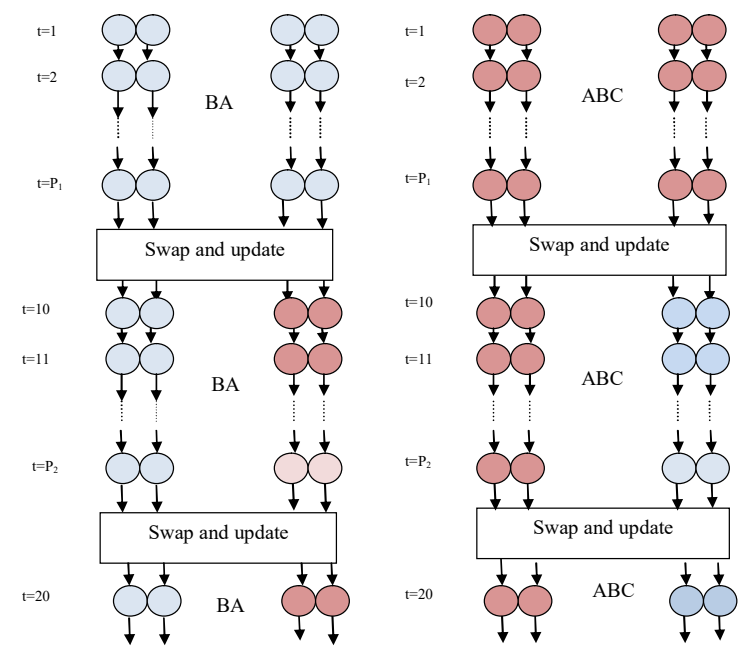

Fig. 1. Diagrammatic representation of the proposed HABC-BA algorithm

\section{Results and Discussions}

\subsection{Experimental Procedure}

To reveal the efficiency and demonstration of the proposed HABC-BA technique, it is simulated on 33bus and 69-bus RDSs. It is exploited for determining the optimal positioning and sizing of multiple distributed generators to reduce the real energy and power loss. Here, the proposed technique is analyzed with conventional GWO, PSO, and WOA techniques in order to prove efficiency.

\subsection{3- bus RDS}

To exhibit the performance of the proposed HABC-BA technique, a small RDS comprises of 33 buses is contemplated for the experimentation study. Table 1 states the statistical analysis of the proposed and existing approaches on 33 bus system. Here, the proposed technique is $22 \%, 24 \%, 25 \%$ better than the GWO, WOA and PSO methods with respect to the Best case scenario.

Table 2 states the computation time of the proposed technique with conventional techniques on the 33 bus system. Here, the proposed technique is $22 \%$ superior to the GWO, $20 \%$ superior to the WOA and $18 \%$ superior to the PSO techniques.

Table 1. Statistical Analysis of the proposed and conventional algorithms on 33 bus system

\begin{tabular}{|c|c|c|c|c|c|c|c|}
\hline \multirow{2}{*}{ Methods } & \multicolumn{7}{|c|}{ Power loss (KW) } \\
\hline & Best & Worst & Means & Success Rate (\%) & VSI & $\mathrm{CBN}$ & CBV (p.u) \\
\hline GWO & 75.123 & 76.123 & 77.123 & 73.22 & 0.9234 & 32 & 0.8214 \\
\hline WOA & 73.223 & 74.234 & 73.231 & 74.354 & 0.9345 & 33 & 0.8316 \\
\hline PSO & 76.233 & 76.342 & 78.334 & 74.234 & 0.9435 & 29 & 0.8428 \\
\hline Proposed & 72.123 & 73.234 & 72.345 & 72.344 & 0.9123 & 24 & 0.8134 \\
\hline
\end{tabular}

Table 2. Computational time of the proposed and conventional algorithms on 33 bus system

\begin{tabular}{ll}
\hline Methods & Computation time \\
\hline GWO & 32.23 \\
WOA & 33.23 \\
PSO & 29.10 \\
Proposed & 25.32 \\
\hline
\end{tabular}

\subsection{9-bus RDS}

To exhibit the competence of the proposed HABC-BA technique, it is simulated on a medium-size RDS comprises of 69 buses. Table 3 summarizes the statistical analysis of the proposed and conventional 
techniques on the 69 bus system. Here, the proposed technique is $32 \%, 36 \%, 38 \%$ superior to the GWO, WOA and PSO techniques with respect to the Best case scenario.

Table 4 summarizes the computation time of the proposed technique with conventional techniques on the 69 bus system. Here, the proposed technique is $12 \%$ superior to the GWO, 11\% superior to the WOA and $9 \%$ superior to the PSO techniques.

Table 3. Statistical analysis of the proposed and conventional algorithms on 69 bus system

\begin{tabular}{|c|c|c|c|c|c|c|c|}
\hline \multirow{2}{*}{ Methods } & \multicolumn{7}{|c|}{ Power loss (KW) } \\
\hline & Best & Worst & Means & Success Rate (\%) & VSI & $\mathrm{CBN}$ & CBV (p.u) \\
\hline GWO & 95.224 & 81.145 & 83.324 & 65.126 & 0.8675 & 25 & 0.5466 \\
\hline WOA & 92.156 & 82.346 & 82.265 & 66.214 & 0.8578 & 26 & 0.5423 \\
\hline PSO & 96.145 & 86.128 & 84.228 & 64.167 & 0.8476 & 27 & 0.5674 \\
\hline Proposed & 91.156 & 80.456 & 82.126 & 61.354 & 0.8234 & 21 & 0.5123 \\
\hline
\end{tabular}

Table 4. Computational time of the proposed and conventional algorithms on 69 bus system

\begin{tabular}{ll}
\hline Methods & Computation time \\
\hline GWO & 22.64 \\
WOA & 26.14 \\
PSO & 20.15 \\
Proposed & 18.22 \\
\hline
\end{tabular}

\section{Conclusion}

This paper presents a complex combinatorial issue on optimal sizing and positioning of a DG for the reduction of real energy loss and power loss in RDS was examined. Here, the HABC-BA technique was proposed, which was used to decide the optimal sizing and positioning of the DG. Moreover, the proposed technique was applied to medium, small, and large scale DN to show its strength and advantages. Finally, the performance of the proposed method was analyzed with conventional PSO, GWO, and WOA. The overall analysis exhibits the effectiveness and superiority of the proposed technique against conventional techniques.

\section{Compliance with Ethical Standards}

Conflicts of interest: Authors declared that they have no conflict of interest.

Human participants: The conducted research follows the ethical standards and the authors ensured that they have not conducted any studies with human participants or animals.

\section{References}

[1] Satish Kumar Injeti, "A Pareto optimal approach for allocation of distributed generators in radial distribution systems using improved differential search algorithm",Journal of Electrical Systems and Information Technology, vol. 5, no. 3, pp. 908-92, December 2018.

[2] Tri Phuoc Nguyen, Dieu Ngoc Vo, "A novel stochastic fractal search algorithm for optimal allocation of distributed generators in radial distribution systems",Applied Soft Computing, vol. 70, pp. 773-796, September 2018.

[3] Ashokkumar Lakum, Vasundhara Mahajan,"Optimal placement and sizing of multiple active power filters in radial distribution system using grey wolf optimizer in presence of nonlinear distributed generation", Electric Power Systems Research, vol. 173, pp. 281-290, August 2019.

[4] Juan M. Home-Ortiz, Mahdi Pourakbari-Kasmaei, Matti Lehtonen, José Roberto Sanches Mantovani,"Optimal location-allocation of storage devices and renewable-based DG in distribution systems", Electric Power Systems Research, vol. 172, pp. 11-21, July 2019.

[5] Imran Ahmad Quadri, S. Bhowmick, D. Joshi,"A comprehensive technique for optimal allocation of distributed energy resources in radial distribution systems", Applied Energy, vol. 211, pp. 1245-1260, 1 February 2018.

[6] Haifeng HONG, Zhesheng HURuipeng GUO, Jun MA, Jiong TIAN, "Directed graph-based distribution network reconfiguration for operation mode adjustment and service restoration considering distributed generation",Journal of Modern Power Systems and Clean Energy, Volume 5, Issue 1, pp 142-149,January 2017.

[7] Q. Sun, R. Han, H. Zhang, J. Zhou and J. M. Guerrero, "A Multiagent-Based Consensus Algorithm for Distributed Coordinated Control of Distributed Generators in the Energy Internet," IEEE Transactions on Smart Grid, vol. 6, no. 6, pp. 3006-3019, Nov. 2015. 
[8] Kamakshi Prashadini Swain, Mala De,"A novel electrical proximity index for voltage control in smart distribution system", Electric Power Systems Research, Volume 172, Pages 50-62, July 2019.

[9] N. Acharya, P. Mahat, N. Mithulananthan, An analytical approach for DG allocation in primary distribution network, International Journal of Electrical Power and Energy Systems 28 (10), 669-678, 2006.

[10] M. Chakravorty, D. Das, Voltage stability analysis of radial distribution networks, International Journal of Electrical Power and Energy Systems 23 (2), 129-135, 2001.

[11] A. Rekaby, "Directed Artificial Bat Algorithm (DABA) - A new bio-inspired algorithm," 2013 International Conference on Advances in Computing, Communications and Informatics (ICACCI), Mysore, 2013, pp. 12411246.

[12] A Shankar, N Jaisankar, Dynamicity of the scout bee phase for an Artificial Bee Colony for optimized cluster head and network parameters for energy efficient sensor routing", Simulation, vol. 94 (9), pp. 835-847, 2018.

[13] SB Vinay Kumar, PV Rao, Manoj Kumar Singh,"Multi-culture diversity based self adaptive particle swarm optimization for optimal floorplanning",Multiagent and Grid Systems, vol14, no.1, pp.31-65, 2018.

[14] Singh, Gurinderpal \& Jain, V.K. \& Singh, Amanpreet. (2018). A prediction model for the smart Industries, aiding photovoltaic integrated greenhouse system in biogas heating. Computers \& Electrical Engineering. 10.1016/j.compeleceng.2017.12.042.

[15] RT Satish, KT Reddy, "A technique to find optimal location for wavelet-based image watermarking using genetic algorithm",Machine Graphics \& Vision International Journal,vol. 20 (2), pp.173-196, 2011.

[16] SKM Shareef, RS Rao, "Optimal reactive power dispatch under unbalanced conditions using hybrid swarm intelligence", Computers \& Electrical Engineering, vol.69, pp.183-193, 2018.

[17] N. Misthulananthan, O. Than, V.P. Le, Distributed generator placement in power distribution system using genetic algorithm to reduce losses, Thammasat International Journal of Science and Techonology 9 (3) (2004) $55-62$.

[18] A. Keane, M. O’Malley, Optimal distributed generation plant mix with novel loss adjustment factors, IEEE Power Engineering Society General Meeting (2006).

[19] A. Borghetti, A mixed-integer linear programming approach for the computation of the minimum-losses radial configuration, IEEE Transactions on Power System 27 (3) (2012) 1264-73.

[20] A.C. Rueda-Medina, J.F. Franco, M.J. Rider, A.P. Feltrin, R. Romero, A mixed-integerlinear programming approach for optimal type, size and allocation of distributed generation in radial distribution systems, Electric Power System Research 97 (2013), 133-143.

[21] T. Gozel, M.H. Hocaoglu, An analytical method for the sizing and sitting of distributed generators in radial systems, Electric Power System Research 79(6) (2009) 912-918.

[22] H. Khan, A.C. Mohammad, Implementation of distributed generation (IDG) algorithm for performance enhancement of distribution feeder under extreme load growth, International Journal of Electrical Power and Energy Systems 32 (9) (2010) 985-97. 